"(C) (C) 2011 IEEE. Personal use of this material is permitted. Permission from IEEE must be obtained for all other uses, in any current or future media, including reprinting/republishing this material for advertising or promotional purposes, creating new collective works, for resale or redistribution to servers or lists, or reuse of any copyrighted component of this work in other works."

The definitive version of this article is available at http://ieeexplore.iee.org

Published as: Medeiros, C.R.; Costa, J.R.; Fernandes, C.A., "Passive UHF RFID Tag for Airport Suitcase Tracking and Identification," Antennas and Wireless Propagation Letters, IEEE, vol.10, pp.123-126, 2011, Doi: http://dx.doi.org/10.1109/LAWP.2011.2112326 


\title{
Passive UHF RFID Tag for Airport Suitcase Tracking and Identification
}

\author{
Carla R. Medeiros, Jorge R. Costa, Senior Member, IEEE, Carlos A. Fernandes, Senior Member, IEEE
}

\begin{abstract}
A passive UHF tag configuration is presented for suitcase identification and tracking in airport handling applications. The proposed tag antenna solution is based on a conformal geometry, consisting of a folded dipole with orthogonal arms, appropriate for integration into the wall of injection moulded suitcases during its fabrication process. The tag antenna is designed for the ALIEN Higgs-2 integrated circuit. Simulated frequency dependence of the proposed UHF passive tag shows adequate performance across the world UHF RFID bands. Maximum tag detection range as well as detection isotropy were measured in several test environments both for a prototype tag attached to a suitcase and for a tag embedded in the suitcase wall. Full detection of the embedded tag was obtained for all suitcase orientations in the tested scenarios.
\end{abstract}

Index Terms- UHF RFID, Tag Antennas, Quasi Omnidirectional Detection, Luggage Tracking.

\section{INTRODUCTION}

$\mathrm{T}$ HE 2010 report from the International Air Transport Association - IATA states that lost baggage costs the air industry more than US\$2.5 billion every year [1], corresponding to over 25 million mishandled bags globally. About $10 \%$ of the mishandlings result from poor bar-code read rates. One initiative in the framework IATA's Baggage Improvement Program, was to evaluate the potential of passive UHF RFID technology to replace barcodes and reduce mishandlings. Pilot RFID implementations in several airports around the world revealed that bag-tag read-success rate improved markedly from around $85-90 \%$ with conventional barcode tagging to over $95 \%$ with RFID [2]-[3], along with cost saving associated to baggage handling time reduction.

RFID is a fast growing technology that enables radio detection and reading of tags associated with items [4], without requiring line-of-sight. RFID tags carry a univocal ID code and a memory that can store relevant baggage tracking information. In the referred pilot airport implementations,

Manuscript received .... This work was partly funded by Fundação para a Ciência e Tecnologia, through project RFID-Local PTDC/EEATEL/102390/2008 and by INOV-Inesc Inovação, Portugal under contract QREN 1613 (Mala Segura).

Carla R. Medeiros, Jorge R. Costa, and Carlos A. Fernandes are with Instituto de Telecomunicações, IST, Av. Rovisco Pais 1, 1049-001 Lisboa, Portugal (phone +351-218418480 fax +351-218418472 e-mail Carla.Medeiros@lx.it.pt).

Jorge R. Costa is also with ISCTE - Instituto Universitário de Lisboa, Departamento de Ciências e Tecnologias da Informação, Av. das Forças Armadas, 1649-026 Lisboa, Portugal. passive RFID tags were attached to the classical bar code labels around the baggage handler. In a few cases, these tags got wrapped during the baggage handling, precluding $100 \%$ reading score [2].

One interesting alternative is to embed the passive RFID tag within each suitcase wall during its manufacturing process. In this way, instead of the usual disposable tag solution, the embedded tag can be reused and reprogrammed at the airport check-in desk for each travel. This solution is fairly robust and less expensive for the airports. Additionally, the new generation of tags offers reasonable memory size, enabling a wealth of new applications that are already being considered like baggage self-service kiosks, real-time baggage tracking services, etc [5].

Common UHF RFID tags for airport trials use typical dipole configurations [6] which however do not ensure the best reading score. The objective of this work is to develop a new dedicated UHF passive tag solution, based on 3D geometry [7], appropriate for embedding into the suitcase wall during the injection molding process, while presenting good omnidirectional detection, acceptable reading range and full operation across the whole UHF RFID to ensure worldwide interoperability. A folded dipole tag configuration has been presented before for placement in sharp $90^{\circ}$ corners of boxes [8]. However, sharp corners are not usual in travel bags, and the said paper does not investigate tag performance for other conformal uses, or for other placements to cope with shadowing and wall embedding constrains. Most importantly, the tag from [8] does not operate in full UHF RFID world band, which prevents world-wide interoperability The design challenge in our present work also includes finding the best tag positioning in the suitcase that minimizes misreading originated by its content, by shadowing from other close or stacked bags or close presence of metal form the bag conveyors or other surfaces.

In a preliminary study, the developed tag was simply attached to the suitcase surface [9]. This letter presents the results from the complete study, with the final test results of a fine tuned RFID tag embedded in the wall of a standard injection molded suitcase, after passing the industrial integration process. Systematic tests were conducted in a lab baggage scenario using empty and filled suitcases, with $100 \%$ reading score. Non-systematic tests were also carried in real airport environment with the same $100 \%$ reading score. These scores were reached with far less reader antennas than in usual RFID implementations [10]. 


\section{TAG ANTENNA CONFIGURATION}

The geometry of the proposed UHF RFID tag is presented in Fig. 1. On its basic configuration, the antenna consists of an L-shaped dipole, where one arm is additionally folded to form a tri-axial structure, Fig. $1 \mathrm{~b}$. The folded dual-arm configuration was chosen to achieve good omnidirectional properties and to be conformal with one of the suitcase corners. The model was simulated and optimized using the transient solver in CST MW Studio ${ }^{\mathrm{TM}}$ [11].

The overall dimensions of the developed UHF tag are presented in Fig. 1a, the total antenna length being about half wavelength, with the biggest length in the $x$-axis. The adopted integrated circuit is the ALIEN Higgs-2 IC [12]. The loop is centered with respect to a $0.9 \mathrm{~cm}$ deep reentrance of the foldable dipole arm (Fig. 1), $1.3 \mathrm{~cm}$ apart from the orthogonal arm. For antenna optimization, the ALIEN chip impedance was modeled as an equivalent circuit formed by a parallel resistance of $150 \Omega$ and a $1.2 \mathrm{pF}$ capacitor, at $865 \mathrm{MHz}$ $Z_{\text {chip }}=15.5-\mathrm{j} 152 \Omega[12]$.
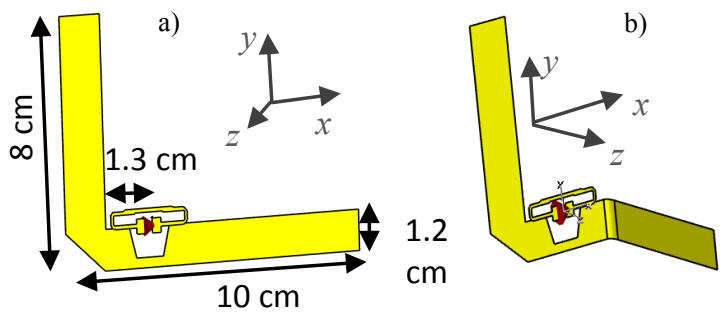

Fig. 1 CST MWS simulation model of the proposed UHF tag: (a) straight "L" configuration; (b) folded configuration.

The prototype tag is printed on an $85 \mu \mathrm{m}$ thick Resist Coated Flex Polyester [13]. To ease lab prototyping, the chip and associated loop were removed from an ALIEN-ALN-9540 - "Squiggle" GEN 2 tag [12] and glued on top of the replica loop from the tag antenna, see Fig. 2a. In this way, the RF signal couples between the loop arms and the tag antenna.

The matching for the RFID tag antenna to the conjugate impedance of the chip was achieved through optimization of the tag arm length and width and by adjusting the loop and slot positions, Fig. 2a.



Fig. 2 (a) Photograph of the proposed tag configuration; (b) photograph of the folded configuration embedded in a transparent part of the suitcase wall.
Fig. 3 shows the simulated frequency behavior of the real and imaginary parts of the antenna impedance for the folded RFID tags at the IC ports. A very good match is obtained to the desired conjugate chip impedance, $Z_{c h i p}=15.5-\mathrm{j} 152 \Omega$ throughout the whole UHF RFID band. At $865 \mathrm{MHz}$, the central frequency of the European UHF RFID sub-band, the folded antenna impedance, is $Z_{a}=15.3+\mathrm{j} 152 \Omega$, including the loop.

The influence of the suitcase material was also evaluated by simulations using CST MW Studio [11]. A straight $150 \mathrm{~mm} \times$ $150 \mathrm{~mm} \times 2 \mathrm{~mm}$ dielectric slab with 2.4 permittivity and 0.003 loss tangent, was added to the folded tag simulation model, directly attached to the tag, to represent the plastic wall of injection molded suitcases.

The power transfer coefficient [4] for the folded RFID tag, based on the fraction of incident power that is transferred to the IC, is shown in Fig. 4. The results are consistent with the antenna impedance curves shown in Fig. 3. In free space the maximum power transfer occurs near $865 \mathrm{MHz}$ and decreases away from this frequency. When attached to a plastic wall, the power transfer curve becomes reasonably flat across most of the international UHF RFID bands (shaded region in Fig. 3) although with decreased level near $90 \%$ due to slight impedance detuning (see Fig. 3). Thus, the proposed RFID UHF tag covers the whole assigned global UHF RFID band, which is a key requirement to allow interoperability between world airports.

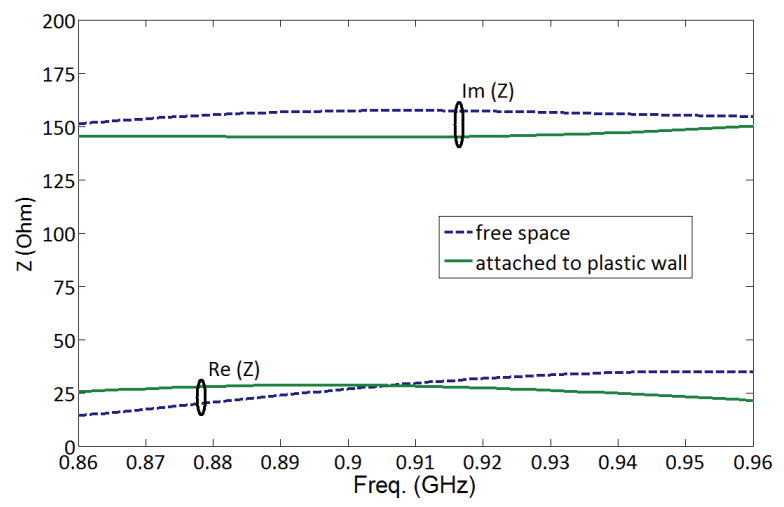

Fig. 3 Simulated frequency dependence of the folded UHF tag impedance in free space and attached to plastic wall.

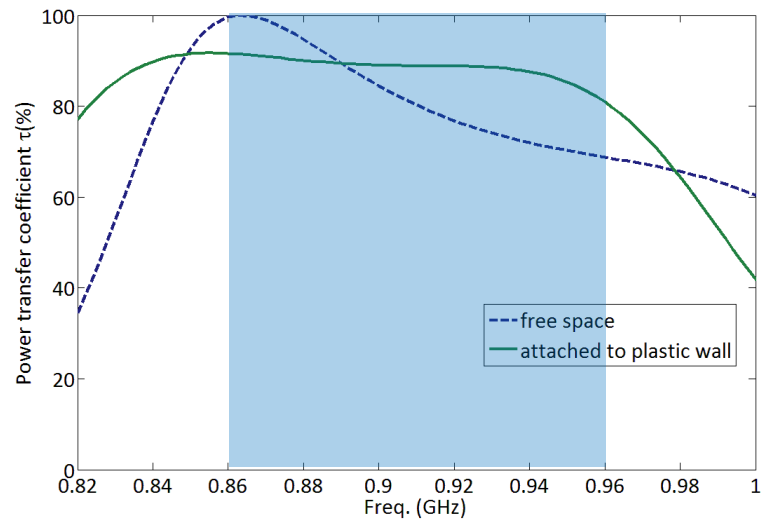

Fig. 4 Simulated Power transfer for the folded UHF tag in free space and attached to plastic wall. The shaded region corresponds to the world assigned RFID band. 
Absolute radiation patterns obtained using CST MW Studio [11] are shown in Fig. 5 for the $x z$ - and $y z$-planes. The results correspond to the tag antenna attached to a plastic wall and demonstrate an almost omnidirectional behavior at both planes. Maximum gain is close to $1.2 \mathrm{dBi}$.

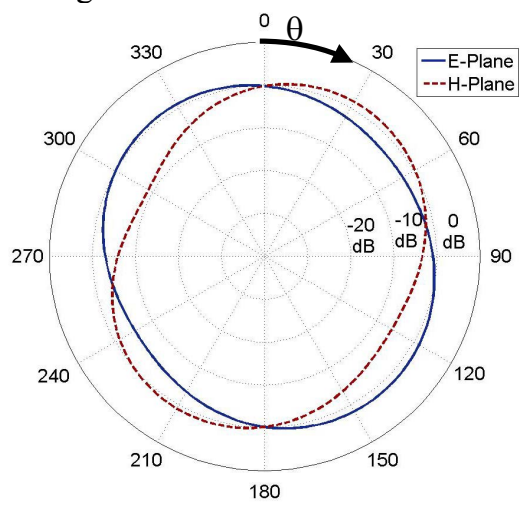

Fig. 5 Simulated radiation pattern gain $(\mathrm{dBi})$ at the $x z$ - and $y z$-planes, for the tag attached to a plastic wall.

\section{EXPERIMENTAL RFID PERFORMANCE}

In order to evaluate the actual UHF RFID performance of the proposed tag for airport baggage application, the tag was embedded in the wall of real 40 Liter suitcases during the injection molding process. This part of the wall was kept transparent (Fig. 2b) to enable checking the position, orientation and integrity of the tag after the industrial process. The tag is positioned near the edge and the arm containing the chip is bent to conform to the suitcase round corner. Tag position and orientation were optimized beforehand to ensure best omnidirectionality, low possibility of obstruction in real scenarios for instance when bags are stacked as usual in airports, and to avoid that possible metallic or liquid items inside the suitcase affect tag performance. Results are compared with the previous case where the tag was simply attached to the wall of a similar suitcase, with the same position and orientation [9]. Tests were performed at 869.25 MHz using the Caen RFID reader A920 Kit [14] with a single commercial circular polarization antenna with $6.5 \mathrm{dBi}$ gain.

Maximum RFID reading range was measured for the suitcase embedded tag and for the suitcase attached tag. The tagged suitcase rested on the floor and the reader antenna was translated $360^{\circ}$ around it at a constant height of $1 \mathrm{~m}$. Measured results are shown in Fig. 6 . In both cases the reading range is typically greater than $3 \mathrm{~m}$ for almost all orientations and reasonably isotropic, suitable for the intended application. There is no significant difference in the results obtained for the two forms of suitcase tagging. The results also agree well with the simulated radiation pattern shown in Fig. 5. Side A in Fig. $6 \mathrm{a}$ corresponds to the $x z$-plane measurement and Side $\mathrm{C}$ in Fig. $6 \mathrm{c}$ corresponds to the $y z$-plane measurement.

Comparison with previous tests using a commercial straight dipole tag (ALIEN - ALN-9540 - "Squiggle" GEN 2) [12] shows that the now proposed tag fills-in some of the previous shadow detection directions. a)

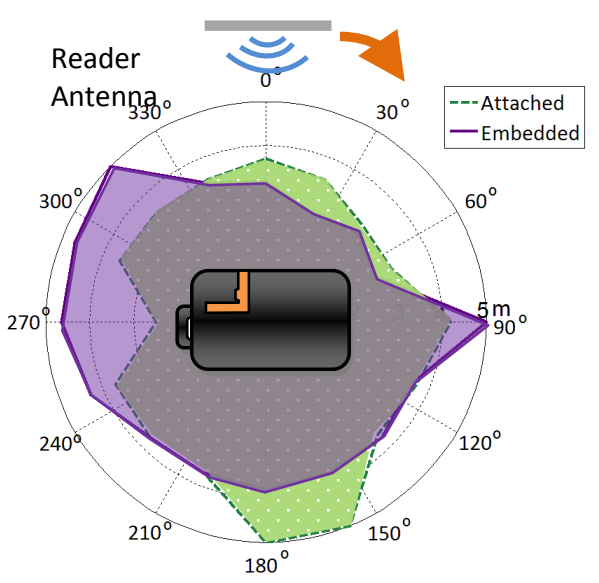

b)

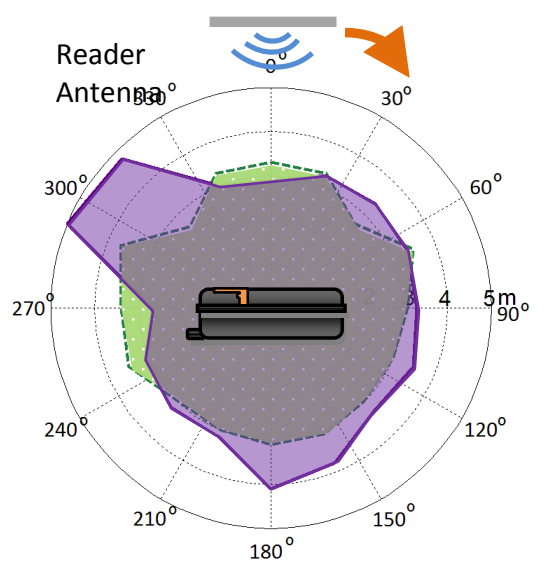

c)

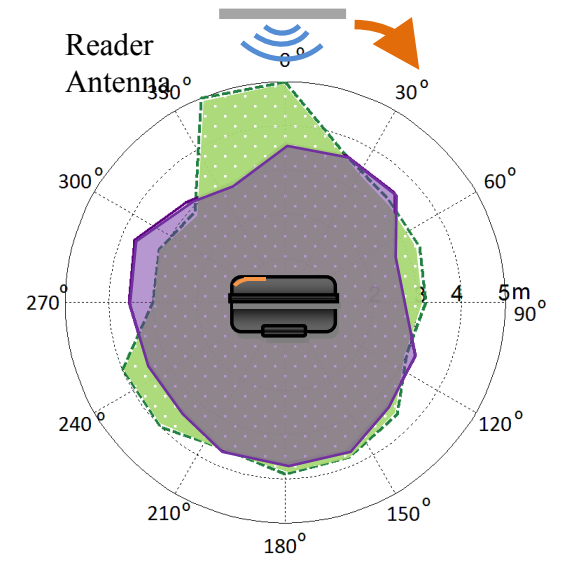

Fig. 6 Top view of maximum reading ranges in m: (a) Side A; (b) Side B; (c) Side C.

The embedded tags were further tested in a lab conveyor belt environment using the same Caen RFID reader A920 Kit [14]. Two circular polarization reader antennas were mounted at each side of the conveyor, $1 \mathrm{~m}$ apart from each other and 30 $\mathrm{cm}$ above the conveyor belt level. An effective alternative would be to use the new embedded antenna concept described in [10] for conveyors, but this falls outside the scope of the present paper. RFID reader counting was recorded while the tagged suitcases were sliding in the conveyor belt for several orientations (Fig. 7). The lab conveyor belt extension is $1.5 \mathrm{~m}$ and the belt slides at an average speed of $0.33 \mathrm{~m} / \mathrm{s}$ over a metallic supporting surface. Tests were conducted both for empty suitcases and when filled with common items, including 
liquids, metal and plastic objects. Results are presented for a single suitcase on the conveyor and for two stacked suitcases.



Fig. 7 Conveyor belt set-up.

TABLE I presents the average reader counts for a single suitcase on the conveyor. The first column indicates the side of the suitcase that is facing up in each case. The results demonstrate very good detection score, always above 5.7 readings/s, corresponding to steady $100 \%$ tag detection. The reading score is hardly affected even when the suitcase is filled with common travel items and the tag is facing down (side $\mathrm{E}$ on TOP) closely contacting with the metal surface beneath the belt.

TABLE I

SINGLE SUITCASE AVERAGE READINGS

\begin{tabular}{|c|c|c|}
\hline Side on TOP & Empty (counts/s) & Filled (counts/s) \\
\hline A & 7.4 & 6.0 \\
\hline B & 7.6 & 8.3 \\
\hline D & 5.7 & 7.5 \\
\hline E & 5.7 & 6.2 \\
\hline
\end{tabular}

Results for stacked suitcases are presented in TABLE II, for different combinations of suitcase orientations. Detection score is again $100 \%$, the minimum of 4.2 counts/s corresponding to a filled suitcase at the bottom of the stack, with the tag closely contacting with the metal surface beneath the belt.

TABLE II

Two-SuITCASE AVERAGE READINGS

\begin{tabular}{|c|c|c|c|c|c|}
\hline \multicolumn{2}{|c|}{ Side on TOP } & \multicolumn{2}{c|}{ Empty (counts/s) } & \multicolumn{2}{c|}{ Filled (counts/s) } \\
\hline $\begin{array}{c}\text { Bottom } \\
\text { Suitcase }\end{array}$ & $\begin{array}{c}\text { Top } \\
\text { Suitcase }\end{array}$ & $\begin{array}{c}\text { Bottom } \\
\text { Suitcase }\end{array}$ & $\begin{array}{c}\text { Top } \\
\text { Suitcase }\end{array}$ & $\begin{array}{c}\text { Bottom } \\
\text { Suitcase }\end{array}$ & $\begin{array}{c}\text { Top } \\
\text { Suitcase }\end{array}$ \\
\hline A & A & 6.1 & 5.6 & 4.7 & 5 \\
\hline E & E & 4.9 & 9.5 & 4.2 & 6.8 \\
\hline E & A & 7.6 & 5.6 & 7.5 & 7.4 \\
\hline A & E & 6.8 & 6.1 & 7.8 & 8.2 \\
\hline
\end{tabular}

It is noted that this excellent score was obtained using only two standard RFID reader antennas covering less than $1 \mathrm{~m}$ section of the conveyor. In a real airport scenario, higher antenna redundancy can be easily implemented to ensure the same reading scores in all difficult cases. Actually nonsystematic tests were carried also in the cargo area of an international airport, where $100 \%$ reading score was confirmed. These results will be presented elsewhere.

\section{CONCLUSION}

A passive folded UHF RFID tag antenna with an orthogonal arm is proposed for embedding into the wall of suitcases, which is adequate for airport automatic luggage tracking and identification. Tag wall embedding ensures tag integrity and enables its reusability in successive user travels, reducing airport costs associated with disposable tags. Results show that the tag radiation pattern is almost omnidirectional when integrated in the suitcase. Co-design of the tag and of its best position and orientation in the suitcase wall contributed for the obtained omnidirectionality and measured detection range of the order of $3 \mathrm{~m}$ in open space.

Experimental tests in lab environment show that the reading score of the proposed tag is $100 \%$ when the suitcases are sliding in a conveyor belt in front of two standard RFID antennas, even when suitcases are filled with travel items and the wall containing the tag is facing down, in the bottom of a two-suitcase stack. The same detection reliability was obtained in a brief non-systematic evaluation at the cargo area of a real airport. Although isolated misreadings can happen for unpredictable arrangements of suitcases in airport conveyors under full service conditions, still the presented results are enough to validate the proposed concept as an alternative to traditional optical barcode labels and disposable RFID tags attached to the bag handle.

\section{ACKNOWLEDGMENT}

The authors thank the fruitful discussions with Prof. António Moreira and with Prof. Mário Nunes who provided the RFID equipment and the bags. The authors also acknowledge the collaboration from Vasco Fred and Carlos Brito for prototype construction, and António Almeida for prototype measurements.

\section{REFERENCES}

[1] (2010) IATA 2010 Annual Report [Online] Available: http://www.iata.org/pressroom/Documents/IATAAnnualReport2010.pdf

[2] (2010) IATA RFID trials for Baggage Tagging [Online] Available: http://www.iata.org/whatwedo/stb/Documents/RFID $\% 20$ trials $\% 20$ for $\%$ 20baggage $\% 20$ tagging.pdf.

[3] (2010) Lyngsoe Systems. [Online] Available: http://www.lyngsoesystems.com/airport/case.asp.

[4] G. Marrocco, "The Art of UHF RFID Antenna Design: ImpedanceMatching and Size-Reduction Techniques," IEEE Antennas and Propagation Magazine, vol. 50, pp. 66-79, 2008.

[5] (2010) SITA - Passenger Self-Service Know-How [Online] Available: http://www.sita.aero/file/2603/passenger-self-service-know-howpositioning-paper-pdf.

[6] K. Rao, P. Nikitin, S. Lam, "Antenna Design for UHF RFID Tags: A Review and a Practical Application", IEEE Transactions on Antennas and Propagation, AP-53, 12, December 2005, pp. 3870-3876.

[7] D. Kholodnyak, P. Turalchuk, A. Mikhailov, S. Dudnikov, and I. Vendik, "3D Antenna for UHF RFID Tags with Eliminated ReadOrientation Sensitivity", in Proc. 36th European Microwave Conference, Manchester, UK, 2006, pp. 583-586.

[8] S. Delichatsios, D. Engels, L. Ukkonen, and L. Sydanheimo, "Albano multidimensional UHF passive RFID tag antenna designs," International Journal of Radio Frequency Identification Technology and Applications, Vol. 1, No. 1, 2006, pp. 24-40. 
[9] C. Medeiros, J. Costa, and C. Fernandes, "UHF Tag for Suitcase RFID Application in Airports, " in Proc. European Conf. on Antennas \& Propagation - EUCAP, Barcelona, Spain, April 2010.

[10] C. Medeiros, J. Costa, and C. Fernandes, "UHF RFID Smart Conveyor Belt with Confined Detection Range," in Proc. IEEE AP-S/URSI International Symp., Charleston, SC, United States, June 2009.

[11] (2010) CST- Computer Simulation Tech. website. [Online]. Available: http://www.cst.com/

[12] (2010) ALIEN Technology website. [Online]. Available: http://www.alientechnology.com.

[13] (2010) Mega Electronics - PCB Laminates. [Online]. Available: http://www.megauk.com/pcb laminates.php.

[14] (2010) CAEN RFID website. [Online]. Available: http://www.caen.it/rfid/. 